\title{
Les nouveaux défis de la santé maternelle et infantile : gestion des systèmes complexes et approche centrée sur la patiente
}

\author{
The new challenges of maternal and child health: complex systems management and the patient- \\ centred approach
}

\author{
B. Dujardin \\ (C) Springer-Verlag France 2012
}

On ne peut que se réjouir que le comité éditorial de la Revue de médecine périnatale aie proposé ce numéro thématique sur la santé internationale. En effet, les articles proposés abordent de nouveaux thèmes souvent peu débattus dans les publications scientifiques cliniques : le financement des programmes de santé, l'évaluation des politiques de santé dans le cadre des Objectifs du millénaire pour le développement (OMD), l'analyse de la situation de la santé maternelle et infantile au Maghreb mais aussi à Cuba.

Pour les spécialistes des politiques et des systèmes de santé, leur quotidien est baigné de concepts tels que : approche systémique, multidisciplinarité, vision globale, systèmes complexes, approche centrée sur les patients ; concepts souvent perçus par les praticiens spécialistes comme étrangers à leur pratique clinique. Ce numéro thématique offre donc une bonne opportunité aux cliniciens et spécialistes de s'ouvrir à de nouveaux horizons.

Cette initiative est d'autant plus importante qu'une des raisons majeures du relatif insuccès de nos politiques de santé en général et en particulier celles concernant la santé maternelle et infantile, est sans doute ce cloisonnement trop strict entre les différents acteurs des systèmes de santé : spécialistes, généralistes, infirmières, sages-femmes, gestionnaires, décideurs et responsables politiques ne parlent pas le même langage et parfois ne se comprennent pas. La situation se complique encore plus si on tient compte des attentes des patients et communautés, acteurs à part entière des systèmes de santé, dont les comportements et les attentes sont trop souvent ignorés ou méconnus par les professionnels de la santé. Sans oublier les spécialistes du management pour améliorer l'organisation des services de santé, ni les économistes pour améliorer l'efficience des soins, ni les politolo-

B. Dujardin $(\bowtie)$

Université Libre de Bruxelles, École de Santé Publique, Centre de recherche « Politiques et Systèmes de santé - Santé Internationale », CP 594, route de Lennik 808, B-1070 Bruxelles, Belgique

e-mail : bruno.dujardin@ulb.ac.be gues pour concevoir de meilleures politiques ; et cette liste d'acteurs n'est certainement pas exhaustive !

Nous devons relever un double défi : promouvoir d'une part une complémentarité et des interactions positives entre les multiples acteurs des systèmes de santé et d'autre part, développer l'approche multidisciplinaire nécessaire pour améliorer de façon pérenne le fonctionnement des services et la qualité des soins. En effet, pour mieux comprendre les comportements de santé des patients et les attentes des différents acteurs d'un système de santé, nous avons besoin d'autres professionnels, notamment de sociologues et d'anthropologues.

Pour mieux illustrer notre propos, citons quelques exemples liés à la santé maternelle et infantile et qui sont valables pour les pays industrialisés comme pour les pays en développement. Ces exemples montrent que de sensibles améliorations en termes de mieux-être des patientes et des familles et de diminution de la morbidité et de la mortalité sont possibles, même sans ressources additionnelles :

- mieux informer les patientes des soins proposés et des alternatives existantes. Ces patientes seront plus régulières et les relations avec les prestataires seront de meilleure qualité ;

- mieux prendre en compte les contraintes socioéconomiques et culturelles que rencontrent un nombre croissant de patientes ;

- discuter et négocier avec les représentants des communautés les mesures concrètes nécessaires pour améliorer l'accessibilité financière, géographique et culturelle ;

- contextualiser nos soins et l'organisation des services de santé pour mieux répondre aux attentes et aux besoins des populations les plus défavorisées ;

- assurer une meilleure transmission de l'information et une meilleure gestion des patientes entre les différents prestataires et entre les différents niveaux du système de soins de santé : évaluer la qualité et l'efficience du processus de référence et de contre référence ; 
- en tant que professionnels, nous centrer sur l'amélioration continue de la qualité des soins, tant du point de vue médical que relationnel et organisationnel ;

- revendiquer et exiger une simplification de l'administration des soins et des services de santé pour nous laisser plus de temps et de disponibilité lors du colloque singulier avec nos patientes et nos communautés ;

- mieux capitaliser nos interventions pour éviter de refaire les mêmes erreurs et assurer la pérennité et la diffusion de nos réussites.

Tous ces exemples, que nous rencontrons presque chaque jour dans notre pratique professionnelle illustrent bien la complexité croissante de nos systèmes de santé et l'importance de promouvoir une approche systémique et globale pour améliorer l'ensemble de leurs déterminants. Quel serait l'intérêt d'avoir des financements plus importants s'ils ne permettent pas d'améliorer l'accessibilité des patients ? Pourquoi améliorer l'accès aux soins si ceux ci sont de piètre qualité ? Comment espérer des soins de qualité optimale si les prestataires de soins travaillent dans de mauvaises conditions et n'ont pas le temps d'écouter et de rencontrer leurs patients ?

Les OMD vont prochainement être rediscutés au sein de l'Organisation des Nations unies. Un nombre croissant d'acteurs proposent que les OMD santé soient fusionnés en un seul, beaucoup plus global : « Assurer la couverture universelle en soins de santé de qualité », la santé maternelle et infantile devenant une des composantes de cet objectif plus général. Cette avancée, si elle se confirme, nous semble tout a fait justifiée et éthique. En effet pourquoi ne se préoccuper de la santé des femmes que lorsque leur utérus est gravide !?! Dans les pays en développement, quel est le sens de sauver une femme grâce à une césarienne de qualité si ensuite, en cas d'appendicite aiguë, elle n'a pas accès à l'acte chirurgical dont elle a un besoin tout aussi urgent ?

Nos patientes, nos communautés ont besoin de systèmes de santé centrés sur leurs besoins mais aussi sur leurs attentes. Pour arriver à ce résultat, les acteurs des systèmes de santé - principalement les prestataires de soins - doivent aussi être reconnus dans leurs besoins et dans leurs attentes pour être à la fois motivés, fiers et heureux d'être des professionnels de santé de qualité !

Notre formation initiale en tant que prestataire de soins, surtout dans le cas des spécialistes, ne nous a pas préparé à la gestion des aspects socioéconomiques, culturels et psychologiques des demandes de santé des patientes. Centrés sur les aspects purement médicaux, nous nous sentons souvent mal à l'aise et maladroits pour gérer la globalité des relations humaines et la complexité des systèmes de santé. Personnellement, après voir commencé ma carrière comme médecin urgentiste dans des camps de réfugiés, j'ai progressivement découvert la santé publique et les systèmes de santé et après 30 ans de vie professionnelle je peux témoigner du « retours sur investissement »: oser la complexité, oser prendre en compte les attentes des patientes apporte beaucoup de satisfaction personnelle et valorise l'estime de soi.

Je vous souhaite une lecture très fructueuse de ce numéro thématique. 